\title{
Exploring Opportunistic Ambient Notifications in the Smart Home to Enhance Quality of Live
}

\author{
Andreas Seiderer, Chi Tai Dang, and Elisabeth André \\ Augsburg University, Universitätsstr. 6a, 86159 Augsburg, Germany \\ seiderer@hcm-lab.de, dang@informatik.uni-augsburg.de, \\ andre@informatik.uni-augsburg.de
}

\begin{abstract}
Smart home technology became more and more widespread within recent years. They aim to make life easier and taking over unwanted tasks. In traditional homes, it is often not clear whether all windows and doors of a house are closed before inhabitants leave the home or go to sleep. However, this can be easily addressed by smart homes. We present a system based on opportunistically located ambient lights that assist inhabitants of a smart home in answering this question. In this paper, we show the concept of our system and the inhabitant-centered design process behind the development. The system was installed in a one-family smart home. We report on insights gained through data collected from this deployment over a period of six months. Within interviews, we found that the ambient light system gave inhabitants a more confident feeling about open doors/windows.
\end{abstract}

Keywords: Ambient Notifications, Ambient Light, Smart Home

\section{Introduction}

"Did you close the window in the bathroom?" Many of us had this situation after just having locked the front door before leaving. Without a smart home, inhabitants of a house would have to unlock the front door again and check the window state. In particular, the chances to oversee opened windows or doors rise with increasing number of windows, doors, and inhabitants of a house. Inadvertently leaving windows or doors open encourages burglars to break in as such houses represent easy targets. Windows or doors that were left opened may also lead to severe damage to the interior of a house caused by water from heavy rain or wind of a strong storm. Smart homes, however, usually have the capability to gather such information through motion detectors and door/window contact sensors and inform their inhabitants before they leave the home or go to bed, thus help avoid windows and doors that were inadvertently left open. Such a functionality exactly fulfills some of the expectations that people have on smart homes, that is, "make life easy" and "taking over the unwanted tasks" [1].

In this paper, we present a system that addresses this circumstance and informs inhabitants about such security risks through ambient notifications in the form of opportunistically located ambient lights. In contrast to LCD displays 
or smartphones that have the capability to show arbitrary textual or graphical content, lights have a limited information channel. Hence, ambient lights are not suitable to directly tell users which of the relevant windows or doors is still open. However, they can be placed unobtrusively in users' periphery and perceived easily $[9,3]$ while having the potential to quickly conveying information of different urgency levels through color, brightness, or effects (e.g. dim loops) [11]. The ambient lights in our system act as a reminder and seek to increase the subjective safety feeling of inhabitants. For example, by glowing with a "good" color (e.g. green) to indicate that all windows and doors are closed, inhabitants receive the signal that it is safe now to leave the home. For the development of our system, we applied an inhabitant-centered design process as every (smart) home is different and habits, as well as suitable opportunistic places for ambient lights, may vary largely. Based on the implemented and deployed system, we collected data from the lights and sensors in a smart home with two inhabitants of a one-family house. We report on insights from the data for a period of six months. In the discussion section, we will report on quantitative insights from the collected data as well as experiences of the inhabitants with the system.

\section{Related work}

Ambient notifications in the form of light colors or light effects received increasing research interest distributed across recent years (e.g., $[2,3,7,5,6,10])$. For example, Hazlewood et al. [3] studied a small spherical object made of frosted glass, which illuminated with different colors in order to reflect the confidence level of students during university courses. Occhialini et al. [8] explored how ambient lights that showed dynamic patterns on the walls of a meeting room help in time management of meetings. Another example for time management was investigated in a lab study by Mueller et al. [7]. Their "Ambient Timer" unobtrusively notified users of upcoming events through ambient light that was emitted by RGB-LEDs mounted behind a monitor. In terms of energy awareness, Gustafsson et al. [2] demonstrated the intuitiveness of their "Power-aware Cord" within a Wizard-of-Oz experiment. Their intention was to increase the awareness of energy consumption of electronic devices by means of glowing light patterns expressed by electrical cords. However, none of the previous works addressed ambient light notifications to opportunistically inform inhabitants of a domestic environment about a "home state".

Domestic environments, such as smart homes, are different from public or work places as domestic environments are private spaces in which acceptance of technology and techniques may not be the same as for public/work spaces. Vastenburg et al. [11] explored the acceptability of notifications in the home and found that acceptability depends on urgency levels of notifications and their intrusiveness in presentation. Acceptance of urgent notifications was high if presented intrusively and acceptance of non-urgent notifications can be increased if presented non-intrusively. 
Pousman et al. [9] presented a taxonomy for 19 existing ambient information systems consisting of four archetypes which they described as symbolic sculptural display, multiple-information consolidators, information monitor display, and high throughput textual display. Our system falls into the category symbolic sculptural display as we display only few information in an abstract sculptural way. Matviienko et al. [6] studied a variety of ambient light patterns and presented design guidelines to help in designing ambient light systems. In particular, their guidelines suggest that colors known from traffic lights (which our system is based on) are most suitable for assessment of everyday situations.

\subsection{Concept of Opportunistic Ambient Notifications}

The concept of our ambient notifications was kept as unobtrusive and simple as possible in order to avoid disturbing inhabitants while enabling them to quickly understand the notification at the first glance. Hence, it is based on opportunistically placed ambient lights, that is, lights embedded into daily routines of inhabitants or daily objects of the household which are part of daily routines as proposed in $[9,10]$. Ambient lights combined with such a placement aim to be placed unobtrusively in users' periphery while at the same time allow inhabitants to quickly perceive and understand notifications in order to support their daily activities. The lights serve to indicate the states of door and window contacts installed in a home. Due to the limited information channel of lights and a potentially high number of door and window contacts, we chose not to encode particular contacts into the notifications in favor of a quickly perceivable accumulated state, which is reflected by three colors known from traffic lights in western countries, that is, green, orange, and red.

Table 1. Color encoding of ambient lights.

\begin{tabular}{|l|l|}
\hline Color & Meaning \\
\hline Green & All critical and non-critical contacts are closed. \\
\hline Orange & At least one non-critical needs to be closed. \\
\hline Red & At least one critical contact must be closed. \\
\hline
\end{tabular}

Table 1 shows our concept's encoding of colors in which we mapped the three colors to an accumulated state that reflects the door/window contact categories critical and non-critical. The critical category represents doors/windows that allow burglars to easily get into a house such as the main door or a window on the first floor. The non-critical category represents, for example, windows on the second floor, which should be closed but would not allow burglars to quickly break into a house (e.g., as there is no direct access from outside). Such windows are often left open in bedrooms for air exchange overnight or to lower humidity in bathrooms. 


\section{Inhabitant-Centered Design Process}

Every building is different (e.g., in terms of architecture, construction, or number of floors, windows, or doors), and every home/household together with its inhabitants has different habits and daily routines. Usually, technology within households is configured to appropriately support such routines as ascertained by Hughes et al. [4]. Inhabitant-centered design denotes the focus of design processes on such daily routines and accompanying experiences instead of addressing tasks and performance/efficiency of tasks known from user-centered considerations. Therefore, an ambient notification system as proposed in this paper must be designed with respect to the particular house as well as an understanding of the daily practices of occupants [11] in order to provide real benefit to inhabitants. Hence, the development of our system followed a design process in which the inhabitants were highly involved during design, implementation, and installation phases.

\subsection{Inhabitants}

For the development of our system, we recruited a household that already had installed home automation technology consisting of motion detectors and contact sensors on all windows and doors as preparation for an alarm system. The onefamily house had three floors including a garden and a garage. The household consisted of two inhabitants aged between 60 and 70 . The inhabitants were not given any fees for their participation but the developed system.

\subsection{Design Process}

Within an initial meeting, we discussed the concept with the inhabitants and made sure that they fully understood the color encodings. Afterwards, we determined situations and daily routines in which the inhabitants particularly have an interest in knowing the state of their windows and doors. We quickly came across daily routines when they are about to leave their home, go to bed, or linger in the living room.

Ambient Light Locations Depending on the determined routines, we identified locations in the building for placement of ambient lights, which opportunistically support their daily routines, for example, locations where the inhabitants often pass by during the routines. Those locations are the front door, the living room, and the bedroom. If inhabitants are located near the front door, they might leave the house and want to make sure that all windows/doors are closed and locked. The ambient light for this routine was installed in the corridor near the front door as there was already a lamp mounted on the wall, c.f. Figure 1a. This lamp was used as an automatic light source at night and also partly enlightened the stairs down to the cellar and up to the second floor. By reusing 

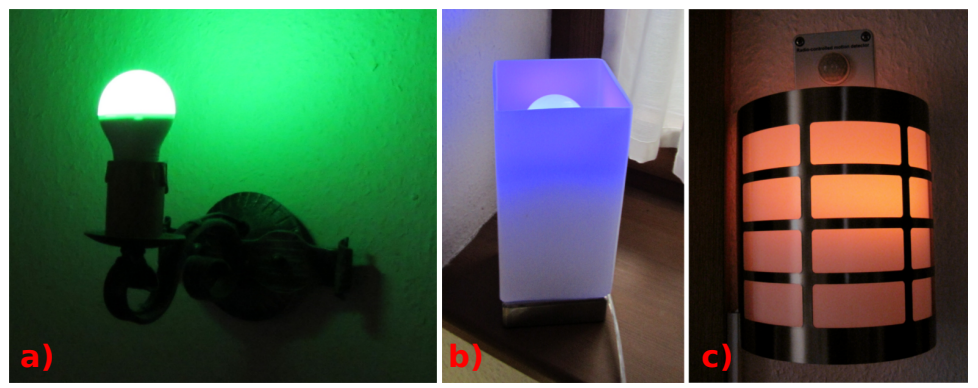

Fig. 1. Picture a) is showing the RGBW bulb near the front door. The lamp in picture b) is placed in the living room. Lamp c) is located near the bedroom. In this case, the wireless motion detector is placed upon the lamp.

the placement and enhancing the lamp to display ambient notifications (i.e., replacing the bulb), the ambient light got an unobtrusive location (as inhabitants were already used to the lamp) and opportunistically support inhabitants in their daily routines.

When the inhabitants linger in the living room for a longer period (e.g. watching TV), they wanted to get notified in case they forgot to close the front door or a window on the first floor. Instead of reusing the main lights on the ceiling for ambient notifications, the inhabitants chose a more unobtrusive location near the terrace, c.f. Figure 1b. Even though this location is in the inhabitants' periphery, the ambient light can still be quickly perceived. Because one of the daily routines in this room is to watch $\mathrm{TV}$, the brightness of the ambient light had to be dimmed down. Otherwise, the ambient light would disturb the daily routines too much.

One of the critical moments when the inhabitants want to make sure that no doors or windows remain open was before they go to sleep. Therefore, the last ambient light was placed near the stairs on the second floor where the inhabitants have their bedroom (c.f. Figure 1c). This ambient light is dimmed down so that not too much light falls into the bedroom but still being bright enough to act as an automatic light for the night, which slightly enlightens the stairs.

Inhabitant-Adapted Color Encodings The final step in the design process was to assign door/window contacts to the categories in Table 1. Depending on the location (and context) of an ambient light, door/window contacts had to be treated slightly different, which must be logical and clear to the inhabitants. The color Green always indicates that all doors and windows are closed and there are no security concerns.

Windows on the second floor are generally assigned to the non-critical category as they do not provide direct access into the building from outside. Their state is indicated through the color Orange if at least one window is open. Inhabitants should get an indication of this state, for example, to prevent damages due to upcoming weather conditions (e.g. rain, wind). There is one exception 
regarding this category. The inhabitants were used to sleep with one window tilted, which should not result in an orange colored warning notifier. Otherwise, the inhabitants would ignore this indication before they go to sleep and probably miss another opened window of the non-critical category. Therefore, the ambient light near the bedroom shows Green if only the window in the bedroom is open.

Red color indicates that a door/window of the critical category is open, for example, the front door or the garage door. Regarding this category, there is an exception for the ambient light near the front door. The state of the front and garage door is not used to adapt the color of this ambient light. Usually, before leaving the house, the garage door is always open and the front door is in direct view, thus, do not need to be indicated by the system. This ambient light is switched to Green if just the garage and/or front door is still open.

The ambient light in the living room behaves like the ambient light near the bedroom with one exception, which was an explicit request expressed by the inhabitants. If only the garage and/or front door is still open, the ambient light in the living room is colored blue as the inhabitants regularly forgot to close this door. In such a case, they wanted a clear and distinct indication of that situation.

\section{System Design}

The existing home automation technology in the smart home of our volunteers employed wireless sensors (door/window contacts, PIR motion detectors) of the home automation system Homematic ${ }^{1}$, which is a widespread technology in Germany. In each room of the chosen ambient light locations, the home had already motion sensors installed. In order to enhance the system with ambient lights as proposed in our concept, we extended the system with radio controlled $(2.4 \mathrm{GHz})$ RGBW LED bulbs, which are part of the Milight ${ }^{2}$ system. They represent a low-cost alternative to the Philips Hue system. Although these bulbs are less expensive, they work quite reliably and produce colored light that has a higher saturation for some colors (especially green) than the Hue bulbs. Nevertheless, the brightness of the Milight bulbs seems to depend more on the color than the Hue bulbs. In our case, the saturation of the color was more important than a stable brightness as the bulbs were just used in addition to existing regular lighting. In order to control the homematic devices as well as the ambient light bulbs, the system made use of so-called bridges as outlined in Figure 2. The existing home automation technology was based on the open source home automation software $\mathrm{FHEM}^{3}$ running on an always-on Linux system powered by a low power Intel NUC-Kit DN2820FYKH with 4 GB RAM and a 120 GB SSD. Hence, we implemented and integrated the ambient light notification logic as an extension of the FHEM installation.

\footnotetext{
${ }^{1}$ http://www.homematic.com/

${ }^{2}$ http://www.milight.com/

${ }^{3}$ http://fhem.de/fhem.html
} 


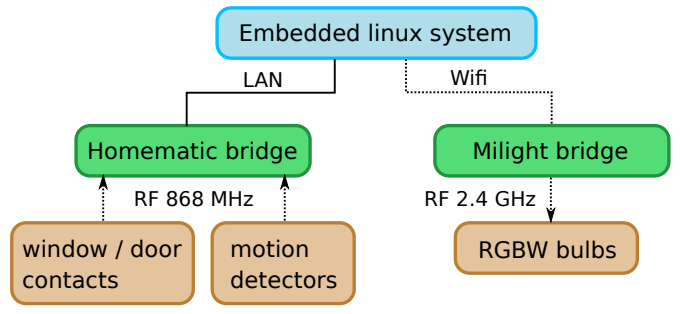

Fig. 2. Overview of the relevant system hardware.

\section{Insights and Discussion}

After designing, implementing, and installing the ambient light system, we collected log data from the home automation system, which included data from all motion sensors and ambient lights. In the following, we analyzed data from a period of six months. Additionally, we did first interviews with the participants.

The system ran quite stable for most of the time and functionality was maintained by an experimenter at least twice a month. Rarely, higher latencies between the detection of an inhabitant and the system output by the ambient lights occurred due to delayed sensor data transmission or higher response time of the LED bulbs. Sometimes, it also happened that signals of the sensors were not received by the home automation, thus an old state was used by the system. If FHEM did not receive the state of sensors for a longer time, the sensor was considered to be "dead", which was identifiable in the logs. For example, this sometimes happened to the contact sensor at the garage door during the winter months. The low temperature condition most probably had a negative (but temporary) influence on the sensor's battery. In order to account for possible transmission errors of the home automation system to the ambient lights, commands (i.e. the accumulated state) to the lights were sent every time motion was detected or the state of a door/window changed.

An important question is how often and when the bulbs were visible to inhabitants and whether the chosen positions for the ambient lights were adequate and actually frequently passed. Information about this can be obtained by analyzing the sensor data from the motion detectors that were placed near the ambient lights. Activity detected through motion sensors resulted in light activity through the ambient lights. The aggregated sum of the detected motion events per hour of the three motion detectors are given in Figure 3. During 8 to 0 o'clock, most of the detected motions occurred in the living room. The second highest event count occurred near the front door. During the night and in the morning from 1 to 7 o'clock, the inhabitants usually passed the bulb near the bedroom. The ambient light in the living room and near the front door are more visible to the inhabitants during the day than the ambient light near the bedroom. In the evening when the inhabitants go to bed and in the morning when they get up the ambient light near the bedroom is more likely to be noticed. 


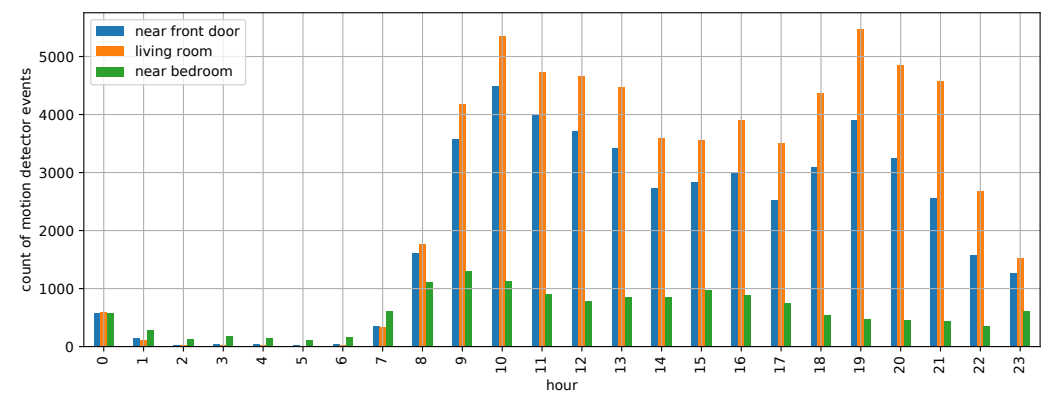

Fig. 3. Count of motion events per hour for each motion detector summed up over six months.

The question whether the system had an influence on the inhabitants cannot be reliably answered by solely interpreting the recorded sensor data. From the collected data, at least two requirements to answer this question can be derived. First, it is possible to detect when the participants left the house by considering a specific time duration without motion during the day as absent. Second, the color of the ambient light that was shown to them before leaving can be identified. However, it is sometimes not clear whether they left specific non-critical windows or doors open by intention or by accident. Furthermore, it would also be possible to detect whether the inhabitants closed doors or windows before they left so that the system would show a green colored light. Still, it is hard to tell if they did this because of the ambient light. In addition, several external influences may occur that change the usual behavior of the inhabitants and are partly visible in the sensor data. This could be a visit from a family member or in case of bad weather for several days.

Thus, we additionally questioned the inhabitants whether the system helped them remember opened doors/windows. One of the inhabitants answered that they usually still know which windows/doors are open but the system gave them a better feeling as the system also confirmed their assumption. Although the lamps cannot show which door/window was open, it was usually sufficient for reminding the inhabitants. In rare cases, it happened that they had to search the open door/window. The inhabitants also confirmed that they were sometimes leaving a (non-critical) window on the second floor open during the day if they were leaving just for a short time or for garden work.

\section{Conclusion}

We have presented the inhabitant-centered design process for an ambient light notification system that addresses smart homes and reported on an ongoing longitudinal deployment of the system. Opportunistic locations for placement of the lights were chosen in tandem with the daily routines of inhabitants. According to interviews with the participants, the system seemed to reduce the amount of forgotten doors/windows but more importantly, an improved certainty about the 
state of the doors/windows was expressed by the inhabitants. Since this system was tested with two inhabitants living in the same home, they also reminded each other. Thus, it would be interesting to test such a system in an additional study with elderly people that live on their own in their own house or apartment.

\section{References}

1. Eggen, B., Hollemans, G., van de Sluis, R.: Exploring and enhancing the home experience. Cognition, Technology \& Work 5(1), 44-54 (2003), http://dx.doi.org/10.1007/s10111-002-0114-7

2. Gustafsson, A., Gyllenswärd, M.: The power-aware cord: Energy awareness through ambient information display. In: CHI '05 Extended Abstracts on Human Factors in Computing Systems. pp. 1423-1426. CHI EA '05, ACM, New York, NY, USA (2005), http://doi.acm.org/10.1145/1056808.1056932

3. Hazlewood, W.R., Stolterman, E., Connelly, K.: Issues in evaluating ambient displays in the wild: Two case studies. In: Proceedings of the SIGCHI Conference on Human Factors in Computing Systems. pp. 877-886. CHI '11, ACM, New York, NY, USA (2011), http://doi.acm.org/10.1145/1978942.1979071

4. Hughes, J., O'Brien, J., Rodden, T., Rouncefield, M., Viller, S.: Patterns of home life: Informing design for domestic environments. Personal Technologies 4(1), 25-38 (2000), http://dx.doi.org/10.1007/BF01613596

5. Mateevitsi, V., Reda, K., Leigh, J., Johnson, A.: The health bar: A persuasive ambient display to improve the office worker's well being. In: Proceedings of the 5th Augmented Human International Conference. pp. 21:1-21:2. AH '14, ACM, New York, NY, USA (2014), http://doi.acm.org/10.1145/2582051.2582072

6. Matviienko, A., Cobus, V., Müller, H., Fortmann, J., Löcken, A., Boll, S., Rauschenberger, M., Timmermann, J., Trappe, C., Heuten, W.: Deriving design guidelines for ambient light systems. In: Proceedings of the 14th International Conference on Mobile and Ubiquitous Multimedia. pp. 267-277. MUM '15, ACM, New York, NY, USA (2015), http://doi.acm.org/10.1145/2836041.2836069

7. Müller, H., Kazakova, A., Pielot, M., Heuten, W., Boll, S.: Ambient Timer - Unobtrusively Reminding Users of Upcoming Tasks with Ambient Light, pp. 211-228. Springer Berlin Heidelberg, Berlin, Heidelberg (2013), http://dx.doi.org/10.1007/978-3-642-40483-2_15

8. Occhialini, V., van Essen, H., Eggen, B.: Design and Evaluation of an Ambient Display to Support Time Management during Meetings, pp. 263-280. Springer Berlin Heidelberg, Berlin, Heidelberg (2011)

9. Pousman, Z., Stasko, J.: A taxonomy of ambient information systems: Four patterns of design. In: Proceedings of the Working Conference on Advanced Visual Interfaces. pp. 67-74. AVI '06, ACM, New York, NY, USA (2006), http://doi.acm.org/10.1145/1133265.1133277

10. Ramos, L., van den Hoven, E., Miller, L.: Designing for the other 'hereafter': When older adults remember about forgetting. In: Proceedings of the 2016 CHI Conference on Human Factors in Computing Systems. pp. 721-732. CHI '16, ACM, New York, NY, USA (2016), http://doi.acm.org/10.1145/2858036.2858162

11. Vastenburg, M.H., Keyson, D.V., de Ridder, H.: Considerate home notification systems: A user study of acceptability of notifications in a livingroom laboratory. Int. J. Hum.-Comput. Stud. 67(9), 814-826 (Sep 2009), http://dx.doi.org/10.1016/j.ijhcs.2009.06.002 